Las cooperativas y el Plan Nacional de Desarrollo K'atun:

Nuestra Guatemala 2032

El camino hacia el triple balance del desarrollo económico, social y ambiental

Yanapanakunakaa K’atun Wiñayniyuka Hatun Suyup Lulayninwan: Guatemalanchik 2032 wata

Pulinakaa kimsapa tinkuynin tinkuy illay wiñayniyuu, achkakunap, llukawsaywan

Ora koperaivapage aike ora Ogenganepage Okibiantagetima K aton asi ora Guatemara 2032

Ora abatsite komantagetokotirokiregipage, timagantsi aike kaninagiterienga

Recepción: 19 septiembre 2020 Corregido: 16 diciembre 2020 Aprobación: 27 febrero 2021

Victor René Ovando Chán

Nacionalidad: Guatemalteca

Universidad de San Carlos de Guatemala

Correo: victorovando22@yahoo.com

ORCID: https://orcid.org/0000-0002-4223-4804

\section{Resumen}

El análisis ha demostrado que las empresas cooperativas, son las que mejor satisfacen todas las dimensiones del desarrollo sostenible, dando respuesta a los problemas económicos, sociales y del medio ambiente. Sin embargo, existen numerosos desafíos que deben enfrentar desde lo institucional, político y trato discriminatorio en la legislación nacional, producto de la falta de comprensión sobre la contribución real y potencial de las cooperativas al cumplimiento de las metas planteadas en el Plan Nacional de Desarrollo K'atun: Nuestra Guatemala 2032. En este contexto, la presente investigación, buscó establecer la correlación intrínseca entre el movimiento cooperativo y el logro de las metas planteadas en el Plan Nacional de Desarrollo K'atun: Guatemala 2032.

\section{Palabras clave:}

cooperativas, desarrollo rural, desarrollo humano integral, incentivación económica, desarrollo sostenible.

\section{Lisichiku limaykuna:}

yanapanakuna, chaklayup wiñaynin, nunap liw wiñaynin, illaywan munachi, takyaa wiñay.

\section{Nibarintsi katingaro:}

koperativa, kaninagitetagantsi jaantagite, kibiagantsi omagaroite, yogagibiagetagani kiregigantsipage, kibiagantsi kaninagiteriengakë

\title{
Datos del autor
}

Victor René Ovando Chán es docente e investigador en educación y medio ambiente. Doctorando en Investigación para el Desarrollo Social por el Centro Universitario de Sur Oriente de la Universidad de San Carlos de Guatemala, Magister en Educación con Orientación en Medio Ambiente por el Centro Universitario de Petén de la Universidad de San Carlos de Guatemala. 


\title{
Cooperatives and the National Development Plan K'atun: Our Guatemala 2032 \\ The path to the triple balance of economic, social and environmental development
}

\begin{abstract}
The analysis has shown that cooperative companies are the ones that best satisfy all dimensions of sustainable development, responding to economic, social and environmental problems. However, there are numerous institutional, political and discriminatory treatment challenges that must be faced in national legislation, as a result of the lack of understanding about the real and potential contribution of cooperatives to the fulfillment of the goals set out in the National Development Plan K'atun: Our Guatemala 2032. In this context, this research sought to establish the intrinsic correlation between the cooperative movement and the achievement of the goals set out in the National Development Plan K’atun: Guatemala 2032.
\end{abstract}

\section{Keywords}

cooperatives, rural development, integral human development, economic incentive, sustainable development.

\section{As cooperativas e o Plano de Desenvolvimento Nacional K'atun: Nossa Guatemala 2032 \\ O caminho para o triplo balanço do desenvolvimento econômico, social e ambiental}

\section{Resumo}

A análise demonstrou que as empresas cooperativas são as que melhor satisfazem todas as dimensões do desenvolvimento sustentável, respondendo aos problemas econômicos, sociais e ambientais. No entanto, são inúmeros os desafios que devem enfrentar desde o institucional, político e o trato discriminatório na legislação nacional, em decorrência da falta de compreensão sobre a real e potencial contribuição das cooperativas para o cumprimento dos objetivos previstos no Plano Nacional de Desenvolvimento K 'atun: Nossa Guatemala 2032. Nesse contexto, esta pesquisa buscou estabelecer a correlação intrínseca entre o movimento cooperativo e o cumprimento das metas estabelecidas no Plano Nacional de Desenvolvimento K'atun: Guatemala 2032.

\section{Palavras-chave:}

cooperativas, desenvolvimento rural, desenvolvimento humano integral, incentivos econômicos, desenvolvimento sustentável. 


\section{Introducción}

Guatemala es un país integrado por 14.9 millones de personas de los cuales, el $46.15 \%$ vive en el área rural, 41.66 son indígenas, con un índice de pobreza del 59.3 y una población económicamente activa de 7.1 millones (INE, 2018), un modelo de gobierno democrático y un sistema político sin ideología definida. En el año 2012, el Consejo Nacional de Desarrollo Urbano y Rural (CONADUR), formuló el Plan Nacional de Desarrollo K'atun: nuestra Guatemala 2032, como una forma de armonizar las 51 políticas públicas con las fuentes que las originaron, tomando como justificación el cumplimiento paulatino y que la gestión de las mismas es parte fundamental del quehacer del Estado.

En este contexto, sobresale el movimiento cooperativo como una respuesta de operativización de las metas planteadas en el Plan K'atun 2032, considerando que estas empresas solidarias son estratégicas para dar respuesta de manera sostenible a los problemas del desarrollo económico, protección del medio ambiente y de equidad social.

En Guatemala, más de 2.2 millones de personas están vinculadas al sector cooperativo, integrados en 1,114 cooperativas activas, las cuales generan más de 235,000 empleos de estos, el $38 \%$ son para mujeres, lo que demuestra la democratización e inclusión económica con enfoque de género, producto de los principios de este tipo de organización; lo que asegura los medios de subsistencia a la población cuando generan más de 3,400 millones de dólares en ingresos anuales, lo que representa el 7.3 del Producto Interno Bruto del país (Agencia EFE, 2013, Programa Mundial de Alimentos de la Organización de las Naciones Unidad, 2012).

De acuerdo con la OIT y ACI (2014), las cooperativas son una forma de empresa sostenible y participativa que tienen como prioridad reducir la pobreza, promover el empleo, fortalecer la seguridad y soberanía alimentaria y hacer un aprovechamiento sustentable de los recursos naturales. Por tanto, el modelo cooperativo está diseñado para contribuir al triple balance de los objetivos económicos, sociales y ambientales, cuyas contribuciones al desarrollo sostenible van mucho más allá de la creación de empleo.

El presente estudio destaca, la contribución de las cooperativas al desarrollo sostenible e invita a debatir y analizar el papel de estas empresas en el cumplimiento del Plan Nacional de Desarrollo K'atun. Sin embargo, es preciso que las cooperativas sean escuchadas y valoradas en el justo aporte de su contribución al desarrollo del país, para alcanzar el potencial como empresas solidarias. De ahí, el aporte del presente estudio, generar un análisis sobre el sector cooperativo, debido a que existe poca información sobre la materia en el contexto de Guatemala, lo cual es una razón de su invisibilización (Alianza Cooperativa Internacional de las Américas, 202ob, p. 4)

Las cooperativas están presentes en los 5 ejes que el Plan K'atun propone, aunque el INACOP limita su intervención en el eje "riqueza para todos y todas". En ese sentido, su amplia experiencia será de gran utilidad para la puesta en práctica de cada uno de ellos, logrando consecuentemente los Objetivos 5) "Igualdad de género" y 17) "Alianza para lograr los objetivos" a los que están articulados dentro del Plan Nacional de Desarrollo. Pues está claro que, las empresas cooperativas son las que mejor satisfacen todas las dimensiones del desarrollo sostenible.

\section{Metodología}

El estudio es de tipo documental, el cual se realizó con el apoyo de fuentes de este carácter, esto es documentos físicos y electrónicos, basado en los aportes del movimiento cooperativo 
al desarrollo rural integral, desarrollo humano, incentivación económica y desarrollo sostenible, haciéndose resúmenes, paráfrasis, comentarios e interpretaciones. En este contexto, se analizaron varias publicaciones en las cuales se observó una relación estrecha entre cooperativismo y el cumplimiento de las metas propuestas en el Plan Nacional de Desarrollo K'atun: Nuestra Guatemala 2032, el cual se encuentra alineado a los Objetivos de Desarrollo Sostenible, propuestos por la Organización de las Naciones Unidas y adoptados por Guatemala en septiembre de 2015.

\section{Desarrollo rural integral}

Las cooperativas en Guatemala, contribuyen de manera positiva al desarrollo social en su conjunto, a través de la generación de empleos e ingresos y la producción de alimentos; determinada muchas veces por las actividades agrícolas, de consumo, comercialización, pesca y el aprovechamiento de los recursos naturales, principalmente en áreas rurales donde se presentan brechas profundas de desigualdad y exclusión social, que ubican a Guatemala, como el segundo país más pobre del continente con una incidencia de pobreza del 70.5\% (Consejo Nacional de Desarrollo Urbano y Rural [CONADUR], 2009 y 2014; CEPAL, 2018).

En el último Censo de Población y Vivienda realizado por el Instituto Nacional de Estadística (INE) (2018), explica que Guatemala está conformada por una población rural del $46.15 \%$ $(6,876,778)$ de un total de $14,901,286$ habitantes; sin embargo, a pesar de tener una población predominantemente urbana (53.85\%), el 54.52\% de los municipios ( 181 de 340 ) tienen una población altamente rural (60\%) de estos 20 superan el 90\% de ruralidad; además el $87.4 \%$ de los lugares poblados en el país $(15,506)$ son rurales ' (CONADUR, 2014, p. 96, 116).

No obstante, esta ruralidad en el país está condicionada por una población que vive en condiciones de pobreza, muchas veces dos y cuatro veces mayor a la que se vive en el área urbana (Fundación Mainel, 2020, p. 5), con una población rural carenciada estimada en 1,4 mil habitantes (CEPAL, 2018, p. 42) y cuyo mayor impacto de acuerdo con la ENCOVI (2014, p.4), se concentra en la población indígena.

Asimismo, Bárcena (2020) afirma que Guatemala presenta una proyección de la pobreza poco alentadora, con valores de 50.5 para la pobreza general y $21.4 \%$ para la pobreza extrema, lo que ubica a la nación como el segundo país con el mayor índice de pobreza en América Latina, solo detrás de Honduras, lo cual coincide con la opinión de la Fundación Mainel (2020, p. 4) cuando afirma que la lenta reducción en la tasa de pobreza, prevé un aumento en más de 175,000 el número de personas que vivirán en pobreza entre 2019 y 2021.

En ese mismo orden de ideas, históricamente la población rural en Guatemala, ha sido excluida y discriminada, producto de los procesos económicos y sociales ocurridos en el pasado, con un modelo de desarrollo basado en la agricultura de subsistencia. En este contexto, existe un amplio consenso entre muchos actores incluida las Naciones Unidas, la Organización Internacional del Trabajo (OIT) y la Alianza Cooperativa Internacional ( $\mathrm{ACl}$ ), sobre que, las cooperativas son el tipo de organización más idóneo para abordar todas las dimensiones del desarrollo rural integral. De hecho hay quienes piensan que el modelo cooperativista puede resolver muchos de los problemas que aquejan al país.

1 De acuerdo con proyecciones poblacionales, se tiene que en el año 2032 el país será $63.7 \%$ urbano y un $36.3 \%$ rural, lo que implica que se incorporará 6.3 millones de personas adicionales a las ciudades (CONADUR, 2014, p. 95), lo cual es un riesgo para la soberanía alimentaria. 
En este sentido, el cooperativismo constituye una estrategia de solución, para contribuir al desarrollo social y productivo del país (Bonilla, 2014, p.68), considerando que este tipo de asociativismo, con alrededor de 1 mil 114 cooperativas afiliadas, "distribuidas en el territorio nacional, generan 118 mil 500 empleos directos y 32 mil indirectos" (López, 2020) y un ingreso calculado en "más de 3 mil 400 millones de dólares en ingresos anuales, lo que representa el 7.3 por ciento del Producto Interno Bruto" (Alianza Cooperativa Internacional de las Américas, 2013; Diario de Centro América, 2016); además de proveer, el 20\% del maíz, el 30\% de frijol y el $40 \%$ de papa (Programa Mundial de Alimentos de la Organización de las Naciones Unidas [WFP-ONU], 2012), entre otros productos de la canasta básica, lo que resalta, el papel fundamental de este tipo de organización, a partir de las cuales, se puede mejorar la producción, su distribución y organización, potencializando la seguridad alimentaria y nutricional de la población del área rural.

El modelo cooperativo, tiene en el mundo contemporáneo, un rol fundamental y un valor incalculable en cuanto a que promueve la inclusión, la equidad y la justicia social, al tiempo que ayuda a reducir la pobreza, generan empleos y permite el acceso a servicios y bienes colectivos básicos. Sin embargo, uno de los aportes esenciales de estas organizaciones, es la producción de alimentos, de hecho hay quienes indican que "las cooperativas agrícolas son una parte importante de la solución para lograr un mundo libre del hambre y la pobreza y su consecuente contribución al cumplimiento de los Objetivos de Desarrollo Sostenibles"(Pelico, 2019).

El sistema cooperativo en Guatemala, entiende que para promover el desarrollo rural integral, se requerirá de una economía rural con orientación a la producción de excedentes, diversificando la producción e impulsando cadenas de valor a través de consorcios empresariales sólidos, que permitan mejorar las condiciones de vida de la población rural. En este sentido, el movimiento cooperativo, se ha integrado y diversificado en sus actividades como estrategia para la permanencia y consolidación cooperativa (ver figura 1).

Figura 1.

Cantidad de cooperativas integrales, especializadas y federadas o asociadas según clase.

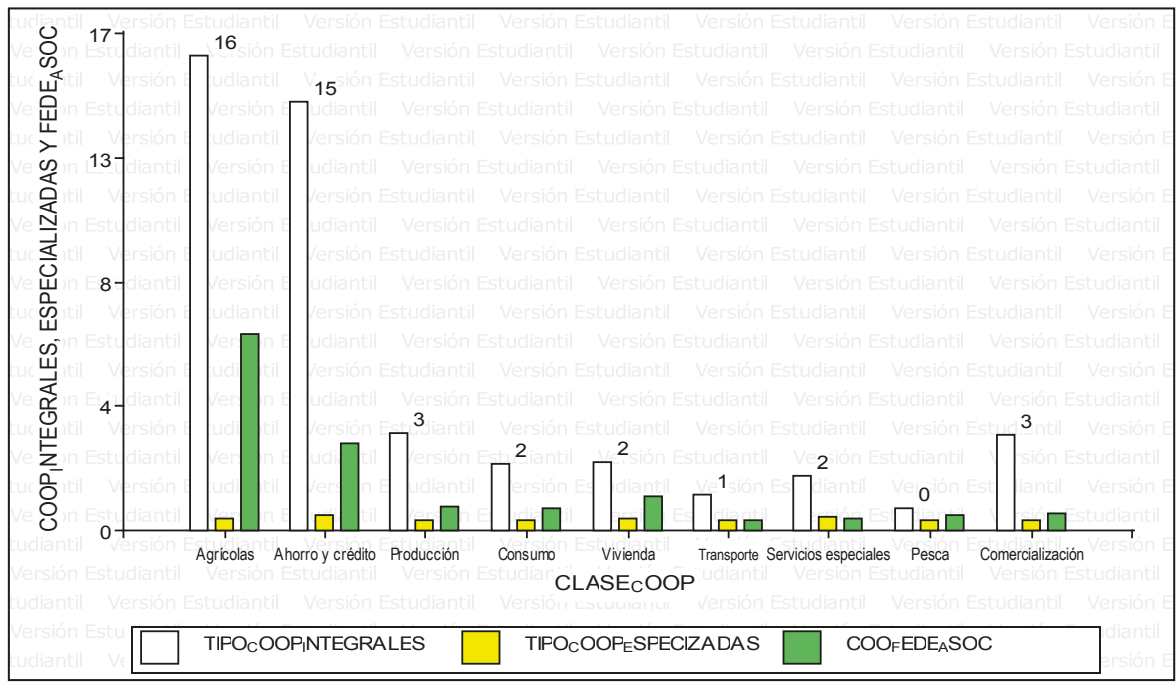

Fuente: Elaboración propia con base en los datos de INACOP (2018). 
Actualmente, ante los constantes embates del modelo económico actual, en un ambiente de libre mercado deshonesto y desigual, las cooperativas han tenido que ampliar sus actividades económicas y diversificarse, como estrategia de resilencia social, por lo que, las cooperativas agrícolas, que tienen mayor presencia en zonas rurales, presentan los mayores niveles de diversificación en sus actividades, con valores de 16 y 15 para las cooperativas de ahorro y crédito, para cada clase según departamento; en tanto que, las cooperativas especializadas son muy pocas, (7 para ser exactos). Asimismo, las cooperativas federadas o asociadas son más evidentes dentro de las clases de cooperativas antes citadas, mostrando para tal caso, 18 federaciones entre ellas: ARTEXCO,RL., FECAIRAN, RL., FECOAR, RL, FECOMERQ, RL., FEDECCON, RL., FEDECOAG, RL, FEDECOAR, RL, FEDECOCAGUA, RL, FEDECOPE, RL., FEDECOVERA, RL, FEDEINCOPAGUA, RL., FEDERURAL, RL, FENACOAC, RL,. FENACOVI, RL., FENAPESCA., FICCI, RL y FINACUP, RL; mientras que dentro de la confederación de cooperativas están CONFECOOP, RL. y CONFECOAC, RL., asimismo, integran asociaciones como: ACODIHUE, ASEDECHI, ASOCUCH, CONAGRAB y GLOBAL FINANZAS, RL.

Por otro lado, las cooperativas no solo han generado fuentes de empleo permanente y temporal, sino que además han cumplido con la Política Laboral de Empleo Digno 2017-2032, garantizando el pleno goce de los derechos laborales de la población rural, campesina e indígena en general y de las mujeres y grupos de personas con capacidades diferentes. Por otro lado, han aportado al crecimiento económico del país, tal es así, que muchas de estas se han organizado en consorcios empresariales ${ }^{2}$, incursionando en nuevos sectores y mercados, tal como lo afirma López y Mora (2012, p.42) y Reyes (2015), convirtiéndose en dueños del 10.08\% de las acciones del grupo financiero BANRURAL, S.A. uno de los bancos más sólidos y con mayor cobertura del sistema bancario en Guatemala (Grupo Financiero Banrural, 2019, p. 4)

De igual manera, una actividad reciente y novedosa en la que las cooperativas intentan participar, es en la generación de energía solar, eólica e hidráulica y las comunicaciones; por consiguiente, la diversificación de las actividades económicas desarrolladas a partir de las cooperativas, "han permitido estabilidad a los impactos y cambios políticos exógenos" mostrando mayor resilencia que cualquier otro tipo de empresa (Álvarez, 2016, p.18). Por lo que, institucional y políticamente debe considerarse al movimiento cooperativo actual, en función de su aporte al desarrollo rural del país (López y Mora, 2012; Segura, O. y Cépedes, 2017).

En este punto del análisis, vale la pena resaltar lo expuesto por Van der Vaeren (2000, p.87) y Valdez (2014, p.106), cuando hacen referencia a que en los años setenta, las cooperativas en Guatemala habían alcanzado un grado de desarrollo social y económico significativo, que parecían confirmarse como alternativa real y viable para los campesinos pobres, lo cual coincide con lo expuesto por la Organización Internacional del Trabajo y la Alianza Cooperativa Internacional $(2014$, p. 2) al afirmar que estas empresas cooperativas aseguran los medios de subsistencia de cerca de la mitad de la población del planeta.

Por tanto, el movimiento cooperativo se define como un dinamizador de la economía, lo cual es sumamente importante en contextos campesinos y rurales, donde se manifiestan los mayores problemas sociales y de atraso del país, con altos índices de pobreza y vulnerabilidad ambiental, por lo que, desde el punto de vista sociológico, las cooperativas son constructoras sociales, organizaciones inventadas y creadas por actores sociales como soluciones específicas para resolver los problemas que se generan con el desarrollo del capitalismo salvaje (López, 2020).

2 Ejemplo de ello son: Hortamaya, Inaplasa, Inpasa, en la industria de productos agrícolas; Inforsa, para productos forestales, y Satyc, para servicios de asistencia técnica y capacitación (Reyes, 2015). 
De manera que, las cooperativas constituyen una salida emergente, a la crisis económica que vive el país, motivado por el incremento del comercio y la industria, sobre la base ética, de la ayuda mutua, la democratización, la igualdad, la equidad y la solidaridad, que posibilitan una distribución horizontal de la riqueza y su consecuente correlación, con "un mejoramiento en los índices de desarrollo humano y la reducción de la pobreza” (López y Mora, 2012, pp. 44,45).

La importancia del cooperativismo en Guatemala es tal, que a finales del siglo pasado e inicios del siglo XXI, la Organización Internacional del Trabajo (OIT) (2002) propuso la promoción de las cooperativas, en tanto que la Organización de las Naciones Unidas para la Alimentación (FAO) en 2012a, definió a este movimiento, como una opción de desarrollo económico, político, social e institucional para el país, ya que permite la autodeterminación y la toma de decisiones de los actores involucrados.

Actualmente, las cooperativas agrícolas garantizan la seguridad alimentaria de la población no solo rural, sino, también urbana, considerando que este sector contribuye con la producción agropecuaria para el consumo interno y la exportación con un aporte del " $40 \%$ al Producto Interno Bruto Agrícola del país" (FAO, 2012a , p.6) y que de acuerdo con el III censo cooperativo en 2008 produjeron Q.2, 704, 377,977.00 (INACOP y CONFECOOP, 2009). En este sentido, las zonas rurales, organizadas en cooperativas, están en las condiciones y capacidades de aportar al desarrollo productivo y la sostenibilidad del medio ambiente (Bonilla, 2014).

Esto tampoco significa que el desarrollo rural, deba ser entendido como una transformación de la sociedad rural, al modo de vida urbano, sino, de mejorar el acceso a más y mejores servicios, el aumento y la diversificación de la producción agropecuaria de manera técnica, el acceso a mercados, con el objetivo de que no exista dependencia alimentaria exterior y se pueda mejorar las condiciones de vida de la población rural, partiendo de la filosofía cooperativista de equidad, participación e inclusión (CONADUR, 2014, p. 97).

En este plano de equidad y participación ciudadana, el movimiento cooperativista registra 2, 232, 738 asociados inscritos (14.98\%) de la población nacional según el último censo poblacional, de los cuales 1, 174,951 (54.89\%) son hombres y 965,757 (45.11\%) son mujeres (ver tabla 1), repartidos en 1,041 cooperativas activas, 13 federaciones y 1 confederación de cooperativas (INACOP, 2018). Un análisis entre los años 2008, 2010 y 2017, refiere que el cooperativismo ha tenido un crecimiento promedio del $26.6 \%$, en tanto que, para el año 2019 se tuvo un crecimiento del $15 \%$. Siendo los departamentos con la mejor tasa \% de crecimiento anual, los departamentos de San Marcos, Suchitepéquez, Chimaltenango y Guatemala; en tanto que, los departamentos con la mayor tasa \% de vinculación al movimiento cooperativo son: Totonicapán, Chiquimula, Santa Rosa, Sololá y Zacapa.

Tabla 1.

Socios inscritos, activos e inactivos

$\begin{array}{cccc}\text { Asociados inscritos } & \begin{array}{c}\text { Asociados } \\ \text { masculinos activos }\end{array} & \begin{array}{c}\text { Asociados femeninos } \\ \text { activos }\end{array} & \begin{array}{c}\text { Total socios } \\ \text { activos }\end{array}\end{array}$

$1,174,951$

965,757

$2,140,708$

Fuente: Elaboración propia (2018) con base en los datos de INACOP (2018). 
A todo esto, no se puede obviar que el área rural, presenta una generalizada precariedad en las condiciones de vida de la población, caracterizada por los altos índices de desigualdad en la tenencia y acceso de la tierra; sin embargo, aquellos que han logrado mantener posesión cooperativa de la tierra, han sido más capaces de protegerse de la especulación en el valor de las tierras, lo cual es sumamente importante, al garantizar el acceso a la tierra como un derecho humano (CONADUR, 2014, p. 97; Ybarra et al., 2009, pp. 27,59).

Los Acuerdos de Paz, específicamente los relativos a la Identidad y Derechos de los Pueblos Indígenas, Reasentamiento de las poblaciones desarraigadas por el enfrentamiento armado y el Acuerdo sobre aspectos socioeconómicos y situación agraria, "señala que los temas agrarios y de desarrollo rural, es donde existe principal déficit en el cumplimiento de los Acuerdos de Paz" (Ministerio de Agricultura Ganadería y Alimentación, 2013, p. 2), por cuanto, recomienda la creación y fortalecimiento de los sistemas de participación comunitaria y una vía adecuada para tal fin es el cooperativismo, entendiendo a estas como un modelo para contribuir al desarrollo social y productivo del país desde lo local (Acuerdos de Paz, 1998, p.120).

De esta manera se tiene que, la organización cooperativa es uno de los principales medios que tienen las comunidades rurales para impulsar su desarrollo económico, social, cultural y ambiental. Para ello se deberá buscar desde lo político una adecuada gestión territorial, que tome en cuenta la dinámica demográfica de los territorios y el desarrollo de capacidades de resilencia local, lo que requerirá desarrollar una política de ordenamiento territorial, así como una política de sistemas rurales y de desarrollo y fortalecimiento cooperativo rural.

\section{Desarrollo humano sostenible}

Bienestar para la gente, es el segundo eje del Plan Nacional de Desarrollo K'atun 2032, al cual se haya vinculado el INACOP, a través del movimiento cooperativo. De acuerdo con el CONADUR (2014, p. 147), el bienestar de las personas debe entenderse como "la construcción de medios de vida sostenibles que provean a la población de las condiciones, habilidades, recursos y servicios para ellos y sus familias". En este punto, las cooperativas reducen las brechas de desigualdad y exclusión social, a través del acceso a la alimentación, la salud y la educación, pues permite la creación de medios de vida sostenibles.

De acuerdo con la FAO (2012a), a lo largo de la historia, el alza de precios de los productos agrícolas, ha impactado de manera dramática en la población rural, principalmente a los que compran sus alimentos o a los campesinos pobres, ya que no pueden cargar con un nivel inflacionario alto (4.5\% para 2021) (Ministerio de Finanzas Públicas, 2020, p. 13), pues deben necesariamente efectuar una recomposición de su patrón alimentario en detrimento de la situación nutricional; de ahí que según la Encuesta Nacional de Salud Materno Infantil (ENSMI) 2014-2015, registra un índice de desnutrición crónica en niños menores de cinco años del 53\% para el área rural y del $58 \%$ en la población indígena (Ministerio de Salud Pública y Asistencia Social (MSPAS), Instituto Nacional de Estadística (INE), ICF International, 2017), siendo los grupos campesinos y agricultores los más afectados, lo cual coloca una enorme presión sobre los recursos naturales, lo que afecta la gobernabilidad social.

En este sentido, las cooperativas agrícolas en Guatemala, contribuyen a la producción de granos básicos como maíz, frijol, arroz que constituye la dieta común del guatemalteco (ver tabla 2); mientras que a nivel mundial proveen, el 32\% de la cuota del mercado mundial (OIT y ACI, 2014, p.7). Las cooperativas tampoco han sido indiferentes, a la actual crisis generada por el 
covid-19, manteniendo la cadena de suministros y continuando con el comercio continental de alimentos, que permitan desarrollar la capacidad de los pequeños agricultores de América para aumentar la producción alimentaria(Alianza Cooperativa Internacional de las Américas, 2020a)

Tabla 2.

Actividad principal de las cooperativas agrícolas

\begin{tabular}{|c|c|}
\hline Región & Actividad de producción principal \\
\hline ala & Producción de maíz, frijol, café, piña y otros productos pecuarios \\
\hline $\begin{array}{l}\text { Región II } \\
\text { Alta Verapaz y Baja Verapaz }\end{array}$ & $\begin{array}{l}\text { Producción de café, cardamomo, té, hule, frijol, maíz, cacao, frutas, } \\
\text { ejote francés y otros productos de la región, además de los relativos } \\
\text { a la actividad pecuaria }\end{array}$ \\
\hline $\begin{array}{l}\text { Región III } \\
\text { Zacapa, Chiquimula, Izabal, El } \\
\text { Progreso }\end{array}$ & $\begin{array}{l}\text { Producción agrícola, venta y distribución de los productos, café, } \\
\text { maíz, frijol, naranja, banano, ejote, banano, cardamomo, limón } \\
\text { criollo y otros propios de la región, }\end{array}$ \\
\hline $\begin{array}{l}\text { Región IV } \\
\text { Jutiapa, Jalapa, Santa Rosa }\end{array}$ & $\begin{array}{l}\text { Producción, industrialización y venta de tomate, papa, aguacate, } \\
\text { hortalizas, chile pimiento, cebolla, maicillo, sorgo, maíz, frijol, chile } \\
\text { y otros cultivos de café, además de la pesca de especies marítimas. }\end{array}$ \\
\hline $\begin{array}{l}\text { Región V } \\
\text { Sacatepéquez, Chimaltenango, } \\
\text { Escuintla }\end{array}$ & $\begin{array}{l}\text { Producción agrícola, venta y distribuc } \\
\text { china, ejote francés, café, trigo, hortal } \\
\text { caña, plátano, tabaco, papa y otros prop }\end{array}$ \\
\hline $\begin{array}{l}\text { Región VI } \\
\text { Quetzaltenango, Retalhuleu, } \\
\text { Totonicapán, San Marcos, } \\
\text { Suchitepéquez, Sololá }\end{array}$ & $\begin{array}{l}\text { Producción agrícola, venta y distribución de los productos de maíz, } \\
\text { ajonjolí, chile, papaya, trigo, café, hortalizas, ejote francés, alverja } \\
\text { china, alverja dulce, arroz, papa, brócoli, coliflor, zanahoria, frutales, } \\
\text { cereales cacao, chile pimiento, plátano, hongos, habas, piña, limón } \\
\text { persa, caña de azúcar, hule, banano, cardamomo y otros productos } \\
\text { de la región, además de la pesca y crianza de ganado vacuno. }\end{array}$ \\
\hline $\begin{array}{l}\text { Región VII } \\
\text { Huehuetenango, Quiché }\end{array}$ & $\begin{array}{l}\text { Producción y comercialización de ajo, café, trigo, papa, ajo, alverja } \\
\text { china, miel de abeja, frutales, cardamomo, ejote, soya y hortalizas }\end{array}$ \\
\hline $\begin{array}{l}\text { Región VIII } \\
\text { Petén }\end{array}$ & $\begin{array}{l}\text { Producción, venta y distribución de maíz, frijol, arroz, miel de abeja, } \\
\text { chile habanero, chile dulce, tomate, soya, cítricos, chicle, pimienta } \\
\text { gorda, ajonjolí, maní, pepitoria, piña, plátano, izote pony, chile } \\
\text { cobanero y otros productos de la región }\end{array}$ \\
\hline
\end{tabular}

Fuente: Elaboración propia, con base en los datos de INACOP, 2018b.

De manera general, las cooperativas ayudan a incentivar la producción de alimentos, superando desafíos como la falta de infraestructura, equipo, ubicación geográfica, volatilidad en los precios del mercado, transporte, acceso a insumos de calidad, entre otros; ofreciendo a sus socios la compra y comercialización conjunta, tienda de insumos, salidas comerciales y acceso a créditos (OIT y $\mathrm{ACl}, 2014$, p. 8).

Además de ayudar a los pequeños productores a innovar y adaptarse a mercados cambiantes, facilita la participación de los cooperativistas en la toma de decisiones, aumentando la capacidad de negociación, para influir en la formulación de políticas públicas. Su participación en los directorios del Fondo de Tierras, del Consejo de Desarrollo Agropecuario, del Consejo Nacional de Desarrollo de las Exportaciones, de la Comisión Nacional de la Vivienda y del Consejo Nacional de Alfabetización, les permite influir en las políticas públicas agrarias, agropecuarias, de comercio, de vivienda y de educación básica (FAO, 2012ª p.7).

Otro aspecto importante de las cooperativas es que, además de facilitar el acceso a alimentos inocuos, según la OIT y $\mathrm{ACl}(2014$, p.8) ayudan a preservar los cultivos autóctonos 
a través de la agricultura familiar incrementando con ello la seguridad alimentaria. De esta manera, las cooperativas contribuyen a la reducción de la pobreza, la soberanía alimentaria y el manejo sostenible de los recursos naturales (FAO, 2012b, p.4); existiendo según López y Mora (2012. p. 47) una correlación entre departamentos de Guatemala con menores niveles de pobreza y la presencia cuantitativa de cooperativas.

El tipo de servicios que brindan las cooperativas son múltiples (ver figura 2), lo cual lleva a la población de áreas rurales a que se busque opciones más accesibles y de costo razonable para la satisfacción de sus necesidades desde nueve posibilidades entre ellas: las agrícolas, de ahorro y crédito, producción, consumo, vivienda, transporte, servicios especiales, pesca y comercialización.

Figura 2.

Cooperativas inscritas, activas e inactivas según clase.

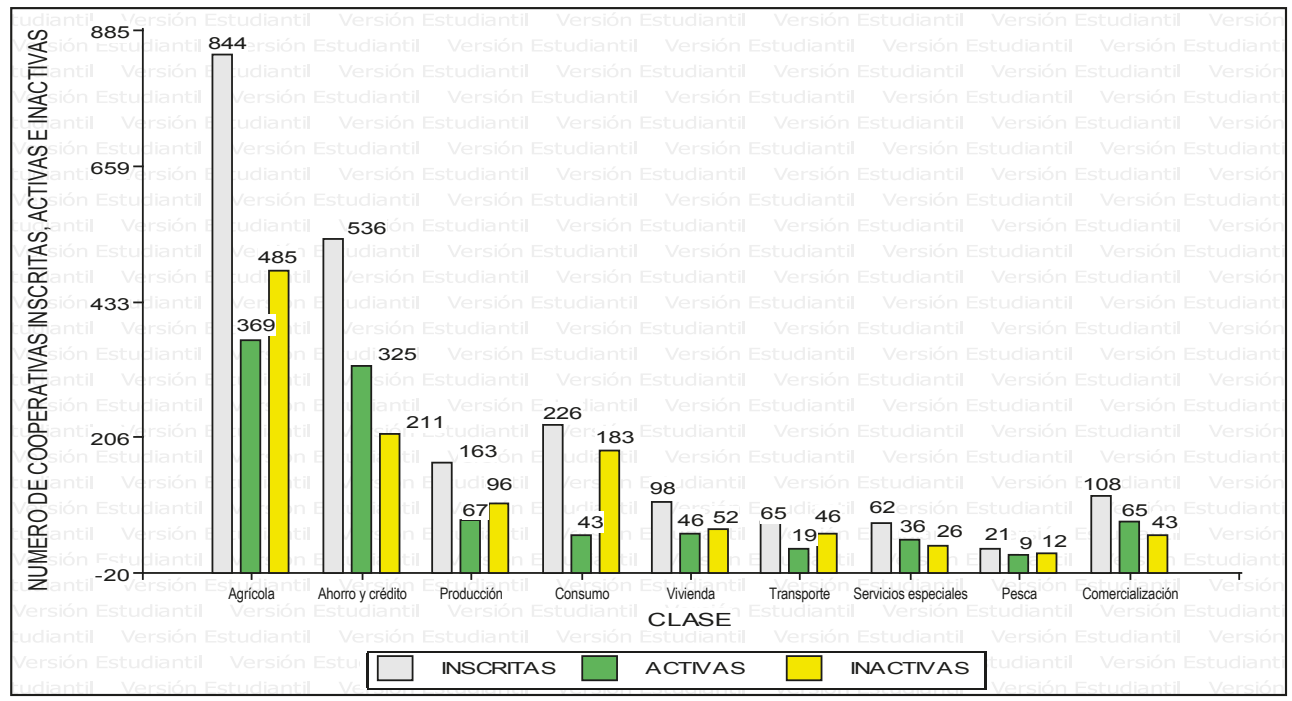

Fuente: Elaboración propia, (2018) con base en los datos de INACOP (2018a).

La salud es el segundo componente del eje "Bienestar para la gente", considerado un derecho humano, que tiene como fin mejorar la calidad de vida de las personas, reduciendo los casos de morbilidad y mortalidad materno-infantil, además de brindar acceso a la salud sexual y reproductiva y de atender a las personas con VIH/SIDA (CONADUR, 2014).

De acuerdo con la Organización Internacional del Trabajo (OIT) y la Alianza Cooperativa Internacional ( $\mathrm{ACl})(2014$, p. 6), las cooperativas, garantizan vidas saludables, mediante la creación de infraestructura para la prestación de servicios de salud, el financiamiento de la asistencia sanitaria y la prestación de servicios de salud en el hogar.

En sentido global, según cifras de la Organización Internacional de Cooperativas de Salud (IHCO), en el mundo existen más de 3, 300 cooperativas de salud en al menos 43 países, permitiendo el acceso a la sanidad a más de 80 millones de personas $(\mathrm{ACl}, 2015)$. En este mismo orden de ideas MacKay (2007, p.140) expone como ejemplo:

Salud-Coop en Colombia, una cooperativa dedicada al cuidado de la salud, es el segundo mayor empleador nacional y sirve al $25 \%$ de la población emergente. En Japón más de 125 
cooperativas médicas sirven a casi 3 millones de pacientes. En Canadá, hay más de 100 cooperativas de salud que prestan principalmente servicios de cuidados en el hogar a más de un millón de personas en ocho provincias

Estos datos avalan la idoneidad del modelo cooperativo en la prestación de servicios de salud y/o asistencia sanitaria y representan para Guatemala una solución a la crisis del sistema de salud, "conciliando los desajustes entre oferta y demanda sanitaria, gestión de costos responsables y calidad de la prestación del servicio, a través de la responsabilidad solidaria de las cooperativas" ( $\mathrm{ACl}, 2015)$.

En Guatemala, las empresas cooperativas de servicios especiales desarrollan actividades diversas, (ver tabla 3), lo cual puede significar una opción altamente viable en el país como se ha dejado entrever.

Tabla 3.

Actividades que desarrollan las cooperativas de servicios especiales en Guatemala.

\begin{tabular}{|c|c|}
\hline Región & Actividad principal \\
\hline $\begin{array}{l}\text { Región I } \\
\text { Guatemala }\end{array}$ & $\begin{array}{l}\text { Servicio de agua potable, tv por cable, servicios educativos y } \\
\text { técnicos, construcción civil, topografía y venta de medicamentos }\end{array}$ \\
\hline $\begin{array}{l}\text { Región II } \\
\text { Alta Verapaz y Baja Verapaz }\end{array}$ & Energía solar, plantas de reciclaje, planes de manejo forestal \\
\hline $\begin{array}{l}\text { Región III } \\
\text { Zacapa, Chiquimula, Izabal, EI } \\
\text { Progreso }\end{array}$ & Construcción de inmuebles, enlonado y sujeción de cargas. \\
\hline $\begin{array}{l}\text { Región IV } \\
\text { Jutiapa, Jalapa, Santa Rosa }\end{array}$ & Sin registro de cooperativas de servicios especiales \\
\hline $\begin{array}{l}\text { Región V } \\
\text { Sacatepéquez, Chimaltenango, } \\
\text { Escuintla }\end{array}$ & Turismo comunitario, operaciones portuarias \\
\hline $\begin{array}{l}\text { Región VI } \\
\text { Quetzaltenango, Retalhuleu, } \\
\text { Totonicapán, San Marcos, } \\
\text { Suchitepéquez, Sololá }\end{array}$ & $\begin{array}{l}\text { Servicio de agua potable, energía eléctrica, turismo, proyectos } \\
\text { educativos }\end{array}$ \\
\hline $\begin{array}{l}\text { Región VII } \\
\text { Huehuetenango, Quiché }\end{array}$ & $\begin{array}{l}\text { Servicio de agua potable, energía eléctrica, alquiler de locales } \\
\text { comerciales, servicios sanitarios, venta de medicamentos }\end{array}$ \\
\hline $\begin{array}{l}\text { Región VIII } \\
\text { Petén }\end{array}$ & $\begin{array}{l}\text { Construcción, reconstrucción, reparación y mantenimiento de } \\
\text { viviendas, actividades afines a la electricidad, plomería, pintura, } \\
\text { herrería, acabados, albañilería y construcción general. }\end{array}$ \\
\hline
\end{tabular}

Fuente: Elaboración propia, con base en los datos de INACOP $2018 \mathrm{~b}$.

El acceso a los servicios prestados a los asociados según López y Mora (2014), por parte de las cooperativas, brinda la posibilidad de tener de manera inmediata, la respuesta a sus necesidades económicas, sociales, culturales, diferente al resto de la población que no está asociada. 
El tercer componente, de bienestar para la gente lo constituye, la educación. Todos saben lo importarte de la educación, para el desarrollo integral de la persona humana, tipificado como un derecho constitucional, las cooperativas favorecen el acceso a este servicio a lo largo de la vida, proporcionando los medios para financiar la educación, lo que se traduce en capacidad para hacer frente a los gastos de educación, además de actuar como centro de enseñanza permanente (OIT y ACI, 2014, p. 6).

En Guatemala, la cobertura educativa en el nivel medio correspondiente al año 2018 fue precario, con un $43.25 \%$ en el ciclo básico y $24.90 \%$ en el diversificado, lo que unido al índice de analfabetismo (12.31\%) que equivale a 1 millón 241 mil 32 guatemaltecos que no saben leer ni escribir (Aceña y Menchú, 2019, p. 13; López, 2017), lo que muestra la incapacidad del gobierno de crear centros escolares que atiendan a este sector de la población, por lo que, es frecuente que las cooperativas hayan creado sus propios establecimientos de enseñanza para impartir educación formal (ver tabla 4).

Tabla 4.

Penetración del modelo cooperativista en la enseñanza

\section{Establecimientos de Enseñanza por Cooperativa \\ Alumnos Cooperativistas \\ Docentes Cooperativistas}

Básico

146,731

Total $\mathbf{1 4 6 , 8 2 4}$
Básico Diversificado

9,867 1,961

Fuente: MINEDUC, 2019.

Estas son entidades no lucrativas que tienen como finalidad, contribuir a la formación integral de los guatemaltecos. En otros casos, el apoyo de las cooperativas ha consistido en invertir un porcentaje de sus beneficios en educación para sus miembros, impartiendo capacitaciones sobre administración contable y financiera, política fiscal, mercadeo, producción, así como programas de alfabetización para los socios, lo cual impacta positivamente en la formación del capital humano de estas organizaciones (INACOP, 2017; FAO, 2012b.).

Según López y Mora (2012, p. 44), la presencia de cooperativas a nivel nacional, evidencia poblados con mayor pujanza económica y con un Índice de Desarrollo Humano (IDH) más elevado, resultado de la dinámica y del volumen de los servicios que se oferta a los asociados. En este sentido, la correlación entre el porcentaje de cooperativas activas y el IDH, es mayor a aquellas zonas geográficas donde existen menos organizaciones de este tipo; por lo que de acuerdo con el Programa de las Naciones Unidas para el Desarrollo (PNUD)(2019, p. 27) (2020, p. 3), la posición de Guatemala en el IDH ha mejorado relativamente, aumentó de 0.481 a 0.663 , un incremento del $37.8 \%$ en sus indicadores, ubicándose en el lugar 127 en el índice de Desarrollo Humano de la PNUD.

\section{Incentivación de la economía nacional}

De acuerdo con el CONADUR (2014, p. 207), el crecimiento económico de Guatemala ha sido irregular durante los últimos años, influenciado por la situación internacional de recesión económica y ralentización, lo que pone en riesgo de llevar al país a una desaceleración o cuan- 
do menos, a un estancamiento económico. No obstante y a pesar de estos problemas, Guatemala ha tenido un desempeño económico sólido en los últimos tres años, con un crecimiento del PIB de 3.1\% en 2016, 2.8\% en 2017 y 3.1\% en 2019, en este sentido se prevé que la economía crezca un $3.3 \%$ en 2019 y $2.8 \%$ en 2020 (Banco Mundial en Guatemala, 2019).

Sin embargo, a esto no se le puede llamar desarrollo, cuando la estabilidad económica no se ha traducido en una aceleración del crecimiento, que permita cerrar la brecha de ingresos con países ricos o cuando la idea de progreso queda limitada al crecimiento económico, pero persisten las altas tasas de pobreza y desigualdad, lo que define un coeficiente de desigualdad para Guatemala del 26.9\% (Gudynas, 2011, p. 22; Banco Mundial en Guatemala, 2019, Programa de las Naciones Unidas para el Desarrollo (PNUD), 2020, p. 4)).

Lo anterior cobra sentido, cuando en Guatemala existe una distribución inequitativa de los medios de producción, la riqueza y los ingresos, donde "la concentración de la riqueza se encuentra en manos del $0.0017 \%$ de la población (26o personas), lo que coincide con la tenencia de la tierra como principal medio de producción, concentrada en el 8\% de los productores, mientras medio millón de familias rurales no poseen tierras para cultivar"(Cañete, 2015, p. 41,44)

Es en este panorama, las cooperativas, se han convertido en un mecanismo de promoción del desarrollo y lucha contra la pobreza. Las actividades económicas y financieras que generan las cooperativas, básicamente están encaminadas a la generación de empleos.

Más de un siglo (116 años) de este modelo de organización en Guatemala, han provocado que las cooperativas posean más de 2.2 millones de socios, generen más de 235,000 empleos y más de 3,400 millones de dólares en ingresos anuales, lo que representa el 7.3 del Producto Interno Bruto del país" (Agencia EFE, 2013, Programa Mundial de Alimentos de la Organización de las Naciones Unidad, 2012).

Según la OIT y ACl (2014, p.10), las empresas cooperativas no solo han soportado mejor los embates de la crisis económica mundial, sino, que también obtienen mejores resultados, consiguen mayores retornos financieros y una mayor productividad, además de empleos más estables. Actualmente las cooperativas existentes en todo el mundo, proporcionan más de 100 millones de empleos, un 20\% más que las empresas multinacionales (FAO, 2012b:7).

Por otra parte, no existen diferencias sustantivas en la calidad del empleo generado por las cooperativas y los que representan los demás sectores formales de la economía donde, se tuvo un salario mínimo para el año 2018 referente a actividades agrícolas y no agrícolas de Q.90.16 diarios, equivalente a Q.2, 742.37 mensuales más una bonificación de Q.250.00 para un total de Q.2, 992.37 (Acuerdo Gubernativo 297-2017). Sin embargo, en el campo cualitativo del empleo generado por estas empresas, existe una notable diferencia, ya que no existe una clásica relación entre obrero-patrono, sino que establecen relaciones más horizontales e igualitarias (López y Mora, 2012, p.44).

En el comercio exterior ha sido importante el aporte de las cooperativas en la evolución económica del país, lo cual evidencia que del total de ingresos por ventas de bienes y servicios, el $24 \%$ proviene de las exportaciones con un total de 655 millones quetzales (ver figura 3 ). 
Figura 3.

Ingreso por venta de bienes y servicios en mercado nacional y de exportación.

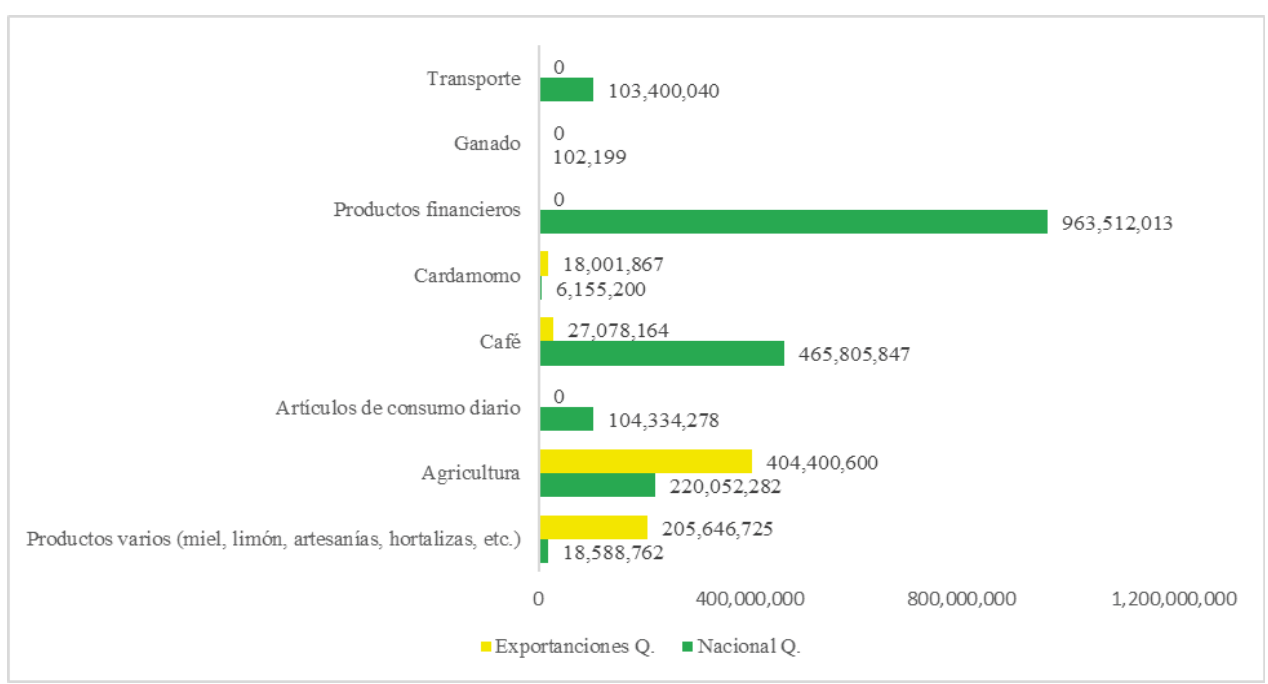

Fuente: Elaboración propia, con base en los datos de INACOP y CONFECOOP, 2008.

Estos datos son promisorios, y se espera aún más cuando las acciones políticas comerciales implementadas, como: la apertura comercial a partir de los compromisos con la Organización Mundial del Comercio (OMC), la integración económica regional y la suscripción de tratados bilaterales, amplíen los mercados en la Unión Europea ${ }^{3}$ (CONDADUR, 2014, p.210).

Asimismo, Guatemala depende mucho de las inversiones y en este punto, las cooperativas han realizado un enorme esfuerzo por tecnificar la producción en el país, de esta cuenta se tiene que, la inversión en plantas industriales equivale a más de 468 millones de quetzales (INACOP y CONFECOOP, 2008).

En la relación con el sector Gobierno, el total de impuestos pagados asciende a 175.4 millones de quetzales, siendo el sector que más contribuye el de ahorro y crédito con 63.3 millones de quetzales (FAO, 2012, p.6). Lamentablemente Guatemala no cuenta con información pública en cuanto a la generación de empleo, así como estimaciones sobre el valor de la producción por parte de las cooperativas (Alianza Cooperativa Internacional de las Américas, 2020b, p. 4), lo que invisibilidad muchas veces su aporte al crecimiento económico del país.

Otro espacio de participación de las cooperativas en la economía guatemalteca, según López y Mora (2012, p. 48), lo constituye el ahorro y el crédito. Dentro de este campo se tiene como dato importante el ahorro juvenil (42\%) lo que demuestra el interés y la confianza por parte de este sector de la población en el sistema cooperativo; mientras que el mayor porcentaje de los créditos va al sector agrícola, (Q.655, 127,356.oo), lo cual devela la relación intrínseca que existe entre las diferentes clases de cooperativas, pues con estos recursos, las cooperativas agrícolas, pueden acceder a los insumos que precisan para sus cultivos; en tanto, que las de consumo les ayuda a comercializar sus productos de tal manera que los socios accedan a productos de la canasta básica bajo condiciones de calidad.

3 En 2008, el movimiento de fondos de las 300 mayores cooperativas, sumó en total 1.1. billones de dólares (FAO, 2012b, p.7). 
Según López y Mora (2012, p. 48), en relación a lo anterior dice: “Las cooperativas son fuente de generación de negocios y empleo, a través de los volúmenes de oferta financiera que maneja a nivel tanto de captación de ahorro como de concesión crediticia", razón por la cual se considera que el sector cooperativo es de suma importancia, para el desarrollo económico del país, especialmente para las áreas rurales, donde se encuentran los sectores más empobrecidos del país, especialmente de origen maya.

\section{Gestión sostenible de los recursos naturales}

Después del lanzamiento del concepto desarrollo sostenible en el Informe de Brundtland en 1987, cuando se concibió como: "aquel desarrollo que satisface las necesidades presentes sin poner en peligro la capacidad de las generaciones futuras para atender sus propias necesidades"; este ha tenido modificaciones y detractores para hacerlo operativo y por considerarlo un oxímoron (Gómez, 2018, p. 16; CONADUR, 2014, p. 239), en este contexto, una de las definiciones más aceptadas en la del Instituto de Agricultura, Recursos Naturales y Medio Ambiente de la Universidad Rafael Landívar (IARNA, URL, 2012), quien lo describe como un proceso de mejoramiento cualitativo y cuantitativo que puede sostenerse en el tiempo, al menos para las dimensión ambiental, económica, social e institucional.

De manera general, de acuerdo con Bonilla (2014, p. 67), "el desarrollo sostenible necesita que las actividades humanas sean armoniosas con los procesos naturales", pues en la medida que se continúe con la degradación de los recursos naturales, se seguirá impidiendo que los avances en la reducción de la pobreza y la seguridad alimentaria se sostengan en el largo plazo (CONADUR, 2014, p. 239).

En 2015 Guatemala adopta oficialmente los Objetivos de Desarrollo Sostenible (ODS) y al mismo tiempo los alineo al Plan Nacional de Desarrollo K'atun: Nuestra Guatemala 2032, lo que permitió un alto nivel de concordancia temática entre ambos instrumentos (Secretaría de Planificación y Programación de la Presidencia (SEGEPLAN), 2017). Atendiendo que las cooperativas por su naturaleza son empresas sensibles a los problemas socioeconómicos y culturales, fundamentadas en valores y principios altamente equitativos y profundamente humanistas, se convierten en aliadas cruciales para conseguir el cumplimiento de estos objetivos(Alianza Cooperativa Internacional de las Américas, 2016; Gutiérrez, 2016).

En este sentido, las cooperativas son vías adecuadas de organización para el desarrollo sustentable, sobre todo, donde el sustento está basado en el manejo y aprovechamiento de los recursos naturales, la agricultura, la agroforestería, la pesca, la agroindustria, el ecoturismo, etc.; lo que podrá impulsar la economía comunitaria desde lo local (Bonilla, 2014, p. 65,69). Es decir, las cooperativas están bien situadas para contribuir al triple balance de objetivos económicos, sociales y ambientales del desarrollo sostenible (Organización Internacional del Trabajo (OIT) y Alianza Cooperativa Internacional (ACI), 2014, p. 2)

Según López y Mora (2012, p. 52), el cuidado del medio ambiente en las actividades cooperativistas, se manifiesta en la preservación del bosque, agua y el suelo; de tal manera, que se han ido convirtiendo en actores cada vez más importantes en lo referente a facilitar el acceso a agua potable y servicio de saneamiento a través de la eliminación de aguas residuales; pues como afirma Díaz (2013, p. 19), las cooperativas revisten una gran importancia en el tema ambiental en correspondencia con el vínculo tan estrecho con el suelo y el agua. 
En la gestión sostenible de los recursos naturales, el manejo y aprovechamiento sostenible de los bosques, ha sido altamente exitoso dentro de las empresas cooperativas campesinas, debido a los réditos monetarios que obtienen. Por lo que, según la OIT y $\mathrm{ACl}(2014$, p. 11), las cooperativas forestales promueven el uso sostenible de las especies tropicales de madera, a través de las concesiones forestales, siendo empresas certificadas por el Consejo de Administración y Forestal (FSC), para operar en el mercado internacional del mueble.

En el caso particular de Guatemala, "las cooperativas son uno de los mayores usuarios del Programa de Incentivos Forestales, -hoy PROBOSQUE- (López y Mora, 2012, p. 52), con lo cual se conservaron entre 1997 y 2014 22,724.59 hectáreas de bosque, correspondiente a 289 proyectos, lo que generó un ingreso de \$13, 027,569.8 dólares y beneficio a 361,021 familias ( Instituto Nacional de Bosques, 2015). Por otra parte, las cooperativas generan cientos de empleos e ingresos en zonas donde existen pocas alternativas de desarrollo económico local; con lo cual se busca mejorar la calidad de vida de la sociedad, a partir de un desarrollo rural sustentable.

Los principios cooperativos, permiten que las cooperativas estén conscientes de su relación con el medio, en este sentido, desempeñan un papel importante en la generación y distribución de energía limpia, estando a la vanguardia en la adopción de fuentes de energía nuevas y renovables, como la solar, eólica e hidráulica (oIT y ACl, 2014, p. 9; López y Mora, 2012). El manejo de los desechos sólidos como el tratamiento de las aguas residuales, tampoco ha sido actividades fuera del orbe cooperativista, tal es así, que en Guatemala, se trabaja en 30 municipios haciendo estas tareas de reciclaje.

Por si esto fuera poco, las cooperativas impulsan pautas de consumo más responsable, diversifican sus actividades, añadiendo la gestión del agua, el turismo ecológico, la producción de alimentos locales de calidad, además de una agricultura orgánica (OIT y ACI, 2014). En otras palabras, "las cooperativas son estratégicas en un proceso de desarrollo rural sostenible, pues se mantienen en el sector con bajas tasas de rentabilidad de capital y son creadas para que los pequeños productores encuentren protección contra los impactos del mercado" (Bonilla, 2014, p. 65).

En síntesis, aunque las cooperativas sean fundamentales para lograr las metas del Plan K'atun 2032 y los Objetivos de Desarrollo Sostenible, no siempre han sido valoradas en el justo peso de su aporte al desarrollo económico, social y ambiental del país, siendo un claro ejemplo, el presupuesto limitado con el que ha funcionado el INACOP durante los últimos 5 años y que en promedio no supera los trece millones de quetzales, además no siempre ha tenido una participación proactiva en los debates de los problemas nacionales e internacionales como ocurrió en el planteamiento de los ODS definidos por la ONU.

\section{Conclusiones}

La población que vive en el área rural de Guatemala, es menor (46.15\%) en comparación a los que viven en áreas urbanas, sin embargo, estas zonas contribuyen de manera positiva a la generación de empleos y a la producción de alimentos, garantizando la seguridad y soberanía alimentaria, pero, necesitan de capacidades organizativas que mejoren la producción y su distribución y una vía adecuada para tal fin, es el cooperativismo.

Las cooperativas ayudan a preservar los cultivos autóctonos, así como la ciencia y la tecnología maya, mejorando con ello el acceso a alimentos inocuos y de buena calidad desde la agricultura familiar. 
Existe una correlación positiva entre departamentos de Guatemala con menores niveles de pobreza y la presencia cuantitativa de las cooperativas, lo cual es coincidente con el porcentaje de cooperativas y el Índice de Desarrollo Humano, siendo menor donde existen un número reducido de organizaciones de este tipo.

Las cooperativas garantizan la educación y salud, mediante la creación de infraestructura para la prestación de estos servicios, su funcionamiento y financiamiento.

Las cooperativas han diversificado sus actividades comerciales, como una forma para que los pequeños productores encuentren protección contra los impactos del modelo económico neoliberal que impulsa un mercado abierto y desleal.

Las cooperativas son importantes para el desarrollo económico del país, ya que son más resilentes a las crisis financieras y económicas a las que constantemente está expuesta la región, producto de la recesión económica y ralentización internacional.

La gestión integral de los recursos naturales mediante el modelo cooperativo, indica que el posible el desarrollo sustentable, mediante la protección y manejo de los bosques, suelos, agua, y el acceso a energía limpia, servicio de saneamiento, entre otros.

El movimiento cooperativo en Guatemala, debe ser un eje estratégico para alcanzar las metas propuestas en el Plan K' atun 2032 y los Objetivos de Desarrollo Sostenible, por tanto, su actuar no se debe limitar al eje riqueza para todos, como supone el INACOP, sino, que debe ser un eje transversal para lograr un verdadero desarrollo sostenible en lo económico, social y ambiental. 
Aceña, M. y W. Menchú (2019). El Sistema Educativo en Guatemala. (Documento final). (Guatemala). Centro de Investigaciones económicas y sociales (CIEN).

Alianza Cooperativa Internacional. (2015). La Organización Internacional de Cooperativas de Salud (IHCO), respalda la propuesta del cooperativismo colombiano para SaludCoop. San José, Costa Rica. Cooperativa de las Américas. [en línea] Consultado 26 de octubre de 2019. https://www. aciamericas.coop/La-Organizacion-Internacional-de-Cooperativas-de-Salud-IHCO-respalda-la

Alianza Cooperativa Internacional de las Américas. (2016). Contribución De Las Cooperativas a Los Objetivos De Desarrollo Sostenible.

Alianza Cooperativa Internacional de las Américas. (2020a). Declaración de Cooperativas de las Américas para el sector agropecuario ante la situación munidal generado por el COVID-19. http:// www.fao.org/fileadmin/user_upload/rlc/docs/covid19/declaracion_ministros_agricultu-

Alianza Cooperativa Internacional de las Américas. (2020b). Mapeo Cooperativo: Datos Estadísticos Informe Nacional: Guatemala Programa Aci-Ue.

Acuerdos de Paz firmados por el Gobierno de la República de Guatemala y la Unidad Revolucionaria Nacional Guatemalteca (URNG). (1998). Guatemala. 3ra. Edición. Universidad Rafael Landívar, Instituto de Investigaciones Económicas y Sociales.

Agencia EFE (2013). Más de un siglo de cooperativismo en Guatemala. Agencia de Negocios y noticas. Economía (en línea). Madrid (España). Consultado 13 agosto 2017. http://www. soy502.com/articulo/mas-de-un-siglo-de-cooperativismo-en-guatemala

Álvarez, A. (2016). Retos de América Latina: Agenda para el Desarrollo Sostenible y Negociaciones del siglo XXI. Problemas del Desarrollo. Revista Latinoamérica de Economía Vol. 47, número 186. Universidad Nacional Autónoma de México. Distrito Federal, México.

Asociación de Abogados y Notarios Mayas de Guatemala. (2017). Minifundio y latifundio, un reflejo de la desigualdad en Guatemala. Guatemala. Boletín Informativo No. 010. NIM-AJPU.

Banco Mundial en Guatemala (2019). Guatemala panorama general. Guatemala. [en línea] consultado el 26 de noviembre de 2019. https://www.bancomundial.org/es/country/ guatemala/overview

Bárcena, A. (2020). El desafío social en tiempos del COVID-19. Consultado el 27 de enero de 2020. https://www.cepal.org/sites/default/files/presentation/files/informe_especial_3_ppt_ covid-19_reporte_3.pdf

Bonilla, G. (2014). Cooperativismo, tierras comunitarias y desarrollo rural. Revista Análisis de la Realidad Nacional. Edición 58, año 3. Guatemala. IPNUSAC.

Cañete, R. (2015). Desigualdad Extrema y Secuestro de la Democracia en América Latina y el Caribe: Privilegios que niegan derechos. In Editora Búho. Consultado el 25 de enero de 2020. https:// www.oxfam.org/sites/www.oxfam.org/files/file_attachments/reporte_iguales-oxfambr.pdf

Comisión Económica para América Latina y el Caribe. (2018a). Ruralidad, hambre y pobreza en América Latina y el Caribe. 68. www.cepal.org/es/suscripciones

Comisión Económica para América Latina y el Caribe. (2018b). Panorama Social de América Latina. LC/PUB.2019/3-P, Santiago de Chile, Chile. ONU.

Consejo Nacional de Desarrollo Urbano y Rural. (2009). Política Nacional de Desarrollo Rural Integral. Guatemala. Conadur/Segeplan. Guatemala.

Consejo Nacional de Desarrollo Urbano y Rural. (2014). Plan Nacional de Desarrollo K'atun Nuestra Guatemala 2032. Guatemala. Conadur/Segeplan.

Confederación de Cooperativas y Federación de Cooperativas Agrícolas. (2010). Propuesta de Desarrollo de la Crisis a la Oportunidad, de la Pobreza a la Acumulación Horizontal de la Riqueza. Una Visión Cooperativa 2012-2037.Guatemala: CONFECOOP y FEDECOAG

Diario de Centro América. (2016, 4 de julio). Cooperativas: un ejercicio de ciudadanía para el cambio - Noticias Última Hora de Guatemala. https://dca.gob.gt/noticias-guatemala-diario-centroamerica/cooperativas-un-ejercicio-de-ciudadania-para-el-cambio/

Díaz, J. (2013). Las Cooperativas y el desarrollo sostenible. Revista Idelcoop, 29(137), 34-50.

Encuesta Nacional de Agropecuaria. (2014). República de Guatemala: Encuesta Nacional Agropecuaria 2014. Guatemala. Instituto Nacional de Estadística. 
Encuesta Nacional de Condiciones de Vida. (2014). República de Guatemala: Encuesta Nacional de Condiciones de Vida 2014. Guatemala. Instituto Nacional de Estadística.

Fundación Mainel. (2020). Estrategia País: Guatemala 2020-2022. Guatemala.

Gómez, B. (2019). El desarrollo sostenible según Brundtland a la sostenibilidad como biomisesis. Bilbao, España. UPV/EHU.

Gutiérrez, M. (2016). Eje 3: Contribución De Las Cooperativas A Los Objetivos De Desarrollo Sostenible. Morelia, México. IV Cumbre Cooperativa de las Américas.

Grupo Financiero Banrural. (2019). Informe De Gobierno Corporativo Grupo Financiero Banrural. Guatemala.

Instituto Nacional de Bosques (INAB) (2015). Programa de fomento al establecimiento, recuperación, restauración, manejo, producción y protección de bosques en Guatemala -PROBOSQUE-. UICN-FAO (Guatemala). Consultado 28 de julio 2020. https://www.marn. gob.gt/Multimedios/1237.pdf

Instituto de Ambiente y Recursos Naturales y Universidad Rafael Landívar. (2012). Perfil Ambiental de Guatemala 2010-2012: Vulnerabilidad local y creciente construcción de riesgo. Guatemala.

Instituto Nacional de Cooperativas. (2018a). Registro de Cooperativas. Unidad de Planificación, programación e informática, INACOOP. (en línea). Guatemala. Consultado 28 de julio 2018. http://inacop.gob.gt/paginas.asp?id=1684\&clc=277

Instituto Nacional de Cooperativas. (2018b). Listado general de cooperativas inscritas por región, departamento y clase. Unidad de Planificación, programación e informática, (Guatemala). INACOOP.

Instituto Nacional de Cooperativas y Confederación de Guatemalteca de Federaciones Cooperativas (2009). III Censo Cooperativo 2008. Las empresas cooperativas enfrentando la crisis, produciendo y generando empleos. Guatemala.

Instituto Nacional de Estadística. (2018). Censo poblacional y habitacional 2018. Guatemala.

López, B. y A. Mora (2012). Visión panorámica del sector cooperativo en Guatemala: un mecanismo de promoción del desarrollo y lucha contra la pobreza. La Paz, Bolivia. OIT, Oficina de la OIT para los Países Andinos.

López, K. (2017, 27 de febrero). MINEDUC. Más de 1 millón de guatemaltecos no saben leer ni escribir. (Guatemala). La Hora.

López, V. (2020). El Cooperativismo Como Un Modelo De Desarrollo S Ocioeconómico Más Humano. Revista FAECO Sapiens, 3 No. 2. Consultado el 27 de enero de 2021. http://portal.amelica. org/ameli/jatsRepo/221/2211235008/html/index.html

López, Y. (2020). Gobierno expresa apoyo al sector de cooperativas - Noticias Última Hora de Guatemala. Diario de Centro América. https://dca.gob.gt/noticias-guatemala-diariocentro-america/gobierno-expresa-apoyo-al-sector-de-cooperativas/

MacKay, L. (2007), "Health cooperatives in BC: The unmet potential”, British Columbia Medical Journal, Vol. 49, No. 3, 139-142

Ministerio de Agricultura Ganadería y Alimentación. (2013). Propuesta De Plan Para Implementar La Política Nacional De Desarrollo Rural Integral-PNDRI-Guatemala, septiembre 2013 i.

Ministerio de Finanzas Públicas. (2020). Informe preliminar: Acerca del "Proyecto de Presupuesto General de Ingresos y Egresos del Estado para el Ejercicio Fiscal 2021, y Multianual 2021- 2025 (Issue 636). http://www.aerocivil.gov.co/autoridad-de-la-aviacion-civil/investigacion/ listado de accidentes 2016/COL 1637 GIA ESP.pdf

Ministerio de Educación, Sistema de Registros Educativos. (2019). Anuario estadístico de la educación de Guatemala. Guatemala. Dirección de Planificación. [en línea] consultado el 25 de enero de 2021. http://estadistica.mineduc.gob.gt/Anuario/home.html\#

Ministerio de Salud Pública y Asistencia Social Instituto Nacional de Estadística, ICF International) (2017). Encuesta Nacional de Salud Materno Infantil 2014-2015. Informe Final. Guatemala, MSPAS/INE/ICF.

Organización Internacional del Trabajo (OIT) y Alianza Cooperativa Internacional (ACI). (2014). Las cooperativas y los Objetivos de Desarrollo Sostenible Debate sobre el desarrollo después de 2015.

Organización de las Naciones Unidas para la Alimentación y la Agricultura. (2012a). Las cooperativas agrícolas alimentan a Guatemala. Guatemala (Guatemala). Día mundial de la alimentación. ONU-FAO 
Organización de las Naciones Unidas para la Alimentación y la Agricultura. (2012b). Las cooperativas agrícolas alimentan al mundo. Roma (Italia). Día mundial de la alimentación. ONU-FAO

Organización Internacional del Trabajo. (2002), "Recomendación 193 sobre la promoción de las cooperativas". Ginebra, Suiza. OIT

Organización Internacional del Trabajo y Alianza Cooperativa Internacional. (2014). Las cooperativas y los objetivos de desarrollo sostenible. Debate sobre el desarrollo después de 2015. Informe de Política. Brucelas, Bélgica

Organismo Ejecutivo (2017, 27 de diciembre). Acuerdo Gubernativo 297-2017. Salarios mínimos para actividades agrícolas, no agrícolas y de la actividad exportadora y de la maquila. Diario de Centro América. No. 72 Tomo CCCVIII viernes 29 de diciembre de 2019.

Pelico, J. (2019). Cooperativas ganan fuerza en A. Latina - Noticias Última Hora de Guatemala. https://dca.gob.gt/noticias-guatemala-diario-centro-america/cooperativas-ganan-fuerzaen-a-latina/

Programa de las Naciones Unidas para el Desarrollo. (2014). Estadísticas-Desarrollo Humano. Guatemala. Índices de Desarrollo Humano y Subíndices. [en línea] Consultado el 13 de noviembre de 2019 https://desarrollohumano.org.gt/estadisticas/estadisticas-desarrollohumano/indice-de-desarrollo-humano-por-departamento-segun-componentes/

Programa de las Naciones Unidas para el Desarrollo. (2016). Más allá del conflicto, luchas por el bienestar. Informe Nacional de Desarrollo Humano 2015/2016. (Guatemala). PNUD.

Programa de las Naciones Unidas para el Desarrollo . (2019). Panorama general Informe sobre Desarrollo Humano 2019. Más allá del ingreso, más allá de los promedios, más allá del presente: Desigualdades del desarrollo humano en el siglo XXI.

Programa de las Naciones Unidas para el Desarrollo . (2020). La próxima frontera: desarrollo humano y el Antropoceno. http://hdr.undp.org/es/data.

Programa Mundial de Alimentos de la Organización de la las Naciones Unidas). (2012). Guatemala: Cooperativas fortalecen la seguridad alimentaria. Guatemala. [en línea] consultado el 1 de noviembre, 2019 https://es.wfp.org/noticias/guatemala-cooperativas-fortalecen-laseguridad-alimentaria

Reyes, S. (2015, 10 de marzo). Cooperativismo. Guatemala. Prensa Libre. https://www.prensalibre. com/opinion/cooperativismo/

Secretaría de Planificación y Programación de la Presidencia SEGEPLAN. (2017). Los Objetivos de Desarrollo Sostenible se alinean al K'atun. Guatemala. Consultado el 19 de enero de 2021. http://elpais.com/elpais/2015/08/07/planeta_futuro/1438937603_140052.html

Segura, O. y Cépedes, J. (2017). Contributions from Cooperatives to Development: Methodological Input for Awareness of the Cooperative Phenomenon (E. S. C \& vol. 104. Vuotto M, Sarria A (eds.). Review of International Co-operation (eds.); Vol. 104).

Universidad Rafael Landívar e Instituto de Agricultura y Recursos Naturales y Ambiente. (2009). Perfil Ambiental de Guatemala 2008-2009: las señales ambientales críticas y su relación con el desarrollo. (Guatemala). URL/IARNA.

Valdez, M. (2014). Territorio y geopolítica: insurgencia y desmovilización en el Petén 1960-2000. Chiapas (México). (Tesis doctoral). Universidad de Ciencias y Artes de Chiapas. Centro de Estudios Superiores de México y Centroamérica.

Van der Vaeren, P. (2000). Perdidos en la selva. Un estudio del proceso de re-arraigo y de desarrollo local de la comunidad Cooperativa Unión Maya Itzá, formada por campesinos guatemalteco, antiguos refugiados, reasentados en el departamento de El Petén. Amsterdam, Holanda. Thela Publisher.

Ybarra, M.; O. Obando; L. Grandia y N. Schwartz. (2010). Tierra, migración y vida en Petén, 19992009. Municipio de Mixco (Guatemala): Instituto de Estudios Agrarios y Rurales -IDEAR-, Coordinación de ONG y Cooperativas -CONGCOOP 